\title{
Analysis of GFDM as a Robust 5G Communication Technique in Noisy Environment
}

\author{
Ferheen Ayaz \\ Department of Engineering and Design \\ University of Sussex \\ Brighton, UK \\ f.ayaz@sussex.ac.uk
}

\author{
Saqib Ayaz \\ Technology Department \\ Workflow Management and Optimization Inc. \\ Somerset, NJ, US
}

\begin{abstract}
One of the challenges of modulation techniques used in Fifth-Generation (5G) is their robustness in noisy environment. Conventional Orthogonal Frequency Division Multiplexing (OFDM) cannot be considered as a $5 \mathrm{G}$ waveform in its original form because of its certain limitations, such as performance degradation by impulsive noise (IN) and high peak to average power ratio (PAPR). Numerous modulation schemes proposed for $5 \mathrm{G}$ communications are able to overcome these drawbacks. Generalised Frequency Division Multiplexing (GFDM) is one of them. This paper analyses the performance of GFDM in presence of Additive White Gaussian Noise (AWGN), IN and Narrow Band Interference (NBI). It is found that GFDM is able to perform better than OFDM and Vector Orthogonal Frequency Division Multiplexing (VOFDM) in presence of noises, which can potentially be present in $5 \mathrm{G}$ applications. Simulation results show that GFDM achieve lower PAPR and Symbol Error Rate (SER) and an average of $10.73 \mathrm{~dB}$ and $4.73 \mathrm{~dB}$ gain in Signal to Noise Ratio (SNR) in presence of IN and combined IN and NBI respectively, as compared to OFDM and VOFDM.
\end{abstract}

Index Terms-GFDM, AWGN, Impulsive Noise, NBI

\section{INTRODUCTION}

Fifth Generation (5G) cellular networks require massive machine type communications (MTC) and support to large scale heterogeneous traffic of Internet of Things (IoT) devices. Therefore, to meet the demands of $5 \mathrm{G}$, research on the physical layer for advanced modulation and multiple access schemes and improved spectral efficiency is being conducted [1]. One of the robust modulation technique of $4 \mathrm{G}$ is Orthogonal Frequency Division Multiplexing (OFDM). It is a type of multicarrier transmission where all subcarriers are orthogonal to each other [2]. Cyclic Prefix (CP) is inserted before OFDM transmission to overcome Intersymbol Interference. It increases communication overhead. Vector-OFDM (VOFDM) was presented as a solution to this overhead. In VOFDM, $K$ consecutive symbols are bolcked together as a vector sequence instead of insertion of CP [3]. On the other hand, Generalised Frequency Division Multiplexing (GFDM) allows CP but it is shortened using a tail biting technique [4] in order to reduce overhead and improve spectral efficiency. This is one of the reasons why GFDM is one of the most acceptable multicarrier transmission techniques for 5G application scenarios [5].

Despite of extensive study on GFDM, its analysis under different kinds of noise is yet to be explored. It has been investigated that the performance of $5 \mathrm{G}$ systems can severely

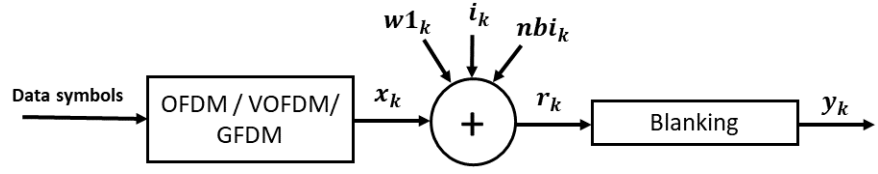

Fig. 1: Addition of noise in the transmitted signal and blanking performed at the received signal.

be degraded by IN [6]. IN consists of randomly occurring short duration pulses with high power. One of the simplest and most commonly used IN mitigation techniques is blanking which nullifies signals with amplitude greater than a certain threshold [7]. Various other IN mitigation techniques have been proposed in literature, but blanking is the simplest and requires low computation. It is also noted that high PAPR of the transmitting signal results in more performance degradation by IN. Therefore, a PAPR reduction technique can be applied at transmitter to make the signal robust against an IN affected channel [8]. Another kind of noise, known as Narrowband Interference (NBI), which has small bandwidth and short duration, also degrades a system's performance [9]. There is a need to investigate GFDM's performance in the presence of both noises to find its practical suitability in 5G.

This paper analyses performance of GFDM system in presence of IN and combined IN and NBI, and evaluates in terms of Signal to Noise Ratio (SNR) and Symbol Error Rate (SER). The contributions of this paper includes performance comparison of OFDM and VOFDM with GFDM in presence of two different kinds of noise to find its practical applicability as a $5 \mathrm{G}$ modulation technique, as well as presenting GFDM as a solution of reducing IN. The organisation of rest of the paper is as follows. Section II describes system and noise modeling. Section III discusses simulation results and conclusion is presented in Section IV.

\section{System AND Noise MODEL}

The transmitted OFDM signal in time domain is obtained by taking Inverse Fourier Transform of the frequency domain signal and is given as

$$
x_{1}(t)=\frac{1}{\sqrt{N}} \sum_{k=0}^{N-1} X_{k} \exp \left(j \frac{2 \pi k t}{T}\right), 0<t<T
$$




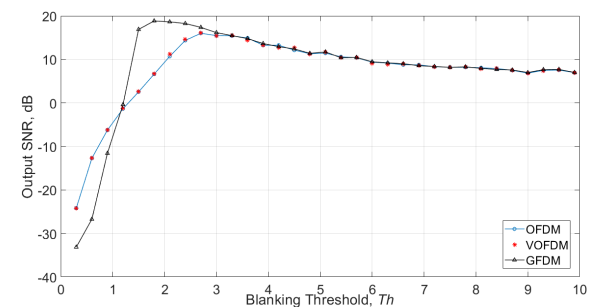

(a) $p=0.01$.

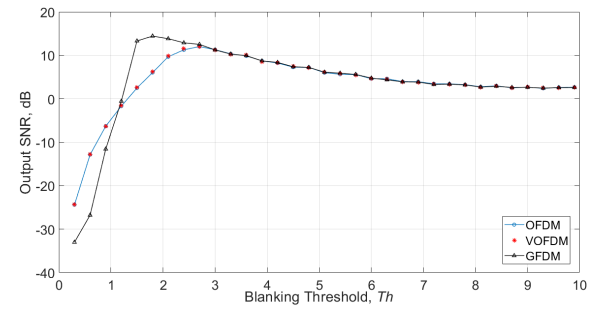

(b) $p=0.03$.

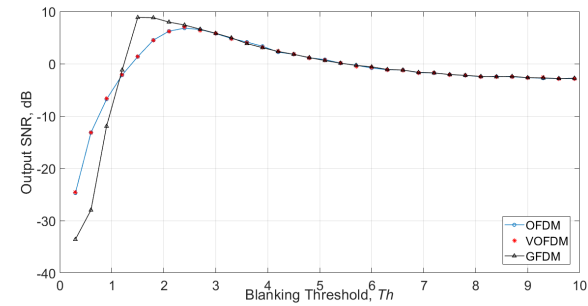

(c) $p=0.1$.

Fig. 2: SNR with only IN affected channel.

where $X_{k}$ denotes the frequency-domain signal, $j=\sqrt{-1}, N$ is number of subcarriers, and $T$ is active symbol interval.

VOFDM divides the length $N$ blocks into $L$ vector blocks, where each vector block (VB) has size $M$, such that $N=$ $L M$. The VB is given as $\boldsymbol{x}_{l}=\left[x_{l}^{M}, x_{l}^{M+1}, \ldots . x_{l}^{M+M+1}\right]^{T}, l=$ $0,1 \ldots, L-1$. VOFDM does VB-wise IFFT, that is,

$$
\bar{x}_{2}(t)=\frac{1}{L} \sum_{l=0}^{L-1} \boldsymbol{x}_{l} \exp \left(j \frac{2 \pi q l}{L}\right), q=0,1, \ldots L-1
$$

where $\bar{x}_{2}(t)$ is a column of vector size $M$.

One GFDM block, $x_{3}(t)$ in time domain is given as

$$
x_{3}(t)=w_{T}(t) \sum_{k=-K / 2}^{K / 2-1} \sum_{m=0}^{M-1} x_{k, m} g T\left(t-m T_{\text {sub }}\right) \exp (j 2 \pi k \Delta f t) \text {, }
$$

where $w_{T}(t)$ is a rectangular window of duration $T, K$ is the unit slot to divide bandwidth $B$, such that $\Delta f=\frac{B}{K}, M$ is the unit slot to divide time duration $T$ such that $T_{\text {sub }}=\frac{T}{M}$, $\Delta f T_{\text {sub }}=1$ and $N=M K \cdot g T(t)$ is the periodic prototype pulse shape and $x_{k, m}$ is the data symbol.

$x(t)$ passed through the channel where Additive White Gauusian Noise (AWGN), IN and NBI are added. AWGN is denoted by $w 1_{k}$ and has variance $\sigma_{w 1}^{2}=(1 / 2) E\left[\left|w 1_{k}\right|^{2}\right]$. IN is modeled as Bernoulli-Gaussian random process as $i_{k}=$ $b 1_{k} w 2_{k}, 0 \leq k \leq N-1$, where $b 1_{k}$ is a Bernoulli process with probability $p$ of $b 1_{k}=1 . w 2_{k}$ is complex zero mean white Gaussian noise with variance $\sigma_{w 2}^{2}=(1 / 2) E\left[\left|w 2_{k}\right|^{2}\right]$. NBI is modeled by Poisson distribution with probability of occurrence $\lambda$, such that, $P(N B I)=\exp (-\lambda) \frac{\lambda^{w 3}}{w 3 !}$, where $\lambda$ is the frequency of occurrence and $w 3$ is the total number of occurrences in a length $N$. NBI is given as, $n b i_{k}=$ $b 2_{k} w 3_{k}, 0 \leq k \leq N-1$, where $b 2_{k}$ is a Binomial process with probability $P(N B I)$ of $b 2_{k}=1$ out of $N$ trials. $w 3_{k}$ is the complex zero mean white Gaussian noise with variance $\sigma_{w 3}^{2}=(1 / 2) E\left[\left|w 3_{k}\right|^{2}\right]$. As shown in Fig. 1, the received signal after addition of noises, is given as $r_{k}=x_{k}+w 1_{k}+i_{k}+n b i_{k}$. Blanking in used to nullify a received symbol if it exceeds a threshold $T h$. The signal after blanking is expressed as

$$
y_{k}= \begin{cases}r_{k}, & \text { if } r_{k} \leq T h \\ 0, & \text { otherwise }\end{cases}
$$

\section{Simulation Results}

The simulation is performed in Matlab, using 64 QAM modulation, with $N=128, \sigma_{w 1}^{2}=1 \times 10^{-5}, \sigma_{w 2}^{2}=10$, $K=64$ and $L=2$. The calculations of SNR, SER and SNR gain are defined in [9]. The average PAPR values of OFDM, VOFDM and GFDM over 100 simulation runs are $24.1725,17.2316$ and 4.5080 respectively. In case of high PAPR, power amplifiers of relatively large linear range are required, otherwise system results in performance degradation. Due to reduced PAPR, GFDM is an efficient, low cost and low power solution for $5 \mathrm{G}$.

Fig. 2 and Fig. 3 show respectively the average SNR and SER of OFDM, VOFDM and GFDM over 100 simulation runs. GFDM outperforms OFDM and VOFDM at all probabilities. However, no difference in performance of OFDM and VOFDM is observed. The maximum SNR and minimum SER is achieved at a specific $T h$, which is lower for GFDM as compared to other schemes. This may be considered as a result of the huge PAPR difference of GFDM and other two schemes. Fig. 4 shows the average SNR of OFDM, VOFDM and GFDM over 100 simulation runs. The maximum SNR is achieved at $\lambda=0.1$ and the minimum SNR is achieved at $\lambda=0.5$. This is because the blanking non-linearity is exclusively a solution to IN mitigation. Therefore, performance degradation is observed with increasing $\lambda$.

Fig. 5 and Fig. 6 show the gain in SNR by GFDM as compared to conventional OFDM. The highest gain occurs at $p=0.01$ with a $13.88 \mathrm{~dB}$ difference for only IN affected channel and $6.95 \mathrm{~dB}$ gain for combined IN and NBI affected channel at $p=0.01$ and $\lambda=0.1$. On an average, the SNR of GFDM is $10.73 \mathrm{~dB}$ and $4.73 \mathrm{~dB}$ higher after passing through only IN and combined IN and NBI affected channel respectively. The SNR gain is not only dependent upon $T h$, but also the probability of occurrence of IN $(p)$ and NBI $(\lambda)$.

\section{CONCLUSION}

The performance of GFDM is analysed and compared with OFDM and VOFDM under individual and combined influence of IN and NBI. As blanking non-linearity is applied at the receiver to mitigate $\mathrm{IN}$, it is observed that the best results of blanking are observed with GFDM with averagely $10.73 \mathrm{~dB}$ higher SNR than conventional OFDM in case of IN only and $4.73 \mathrm{~dB}$ higher SNR with combined IN and NBI. Since blanking is the simplest form of IN mitigation scheme, some 


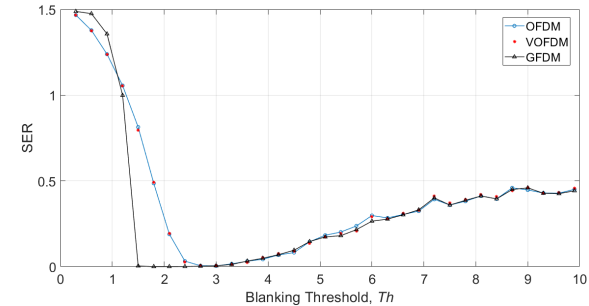

(a) $p=0.01$.

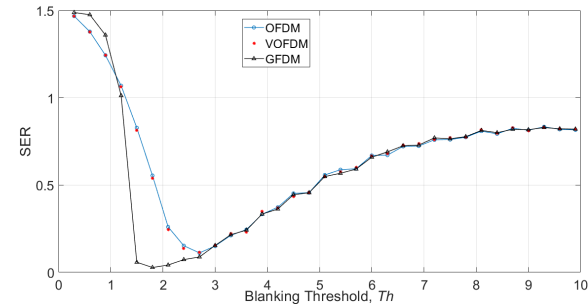

(b) $p=0.03$.

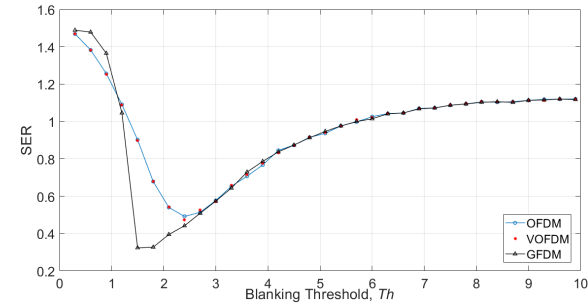

(c) $p=0.1$.

Fig. 3: SER with only IN affected channel.

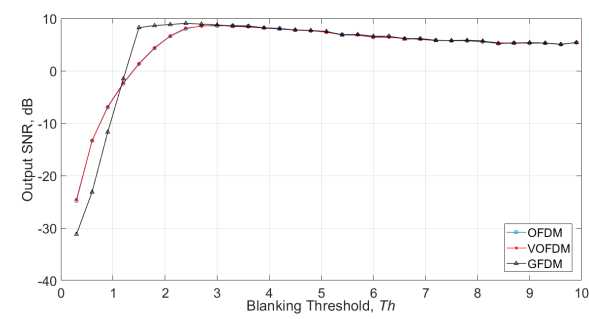

(a) $\lambda=0.1$.

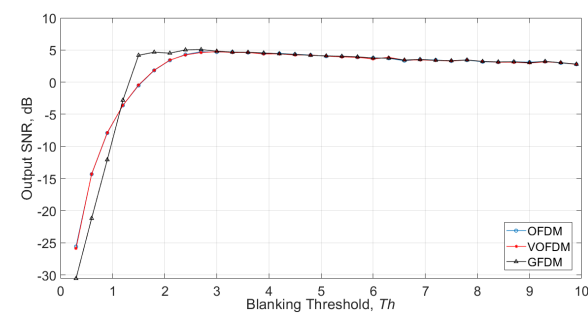

(b) $\lambda=0.25$.

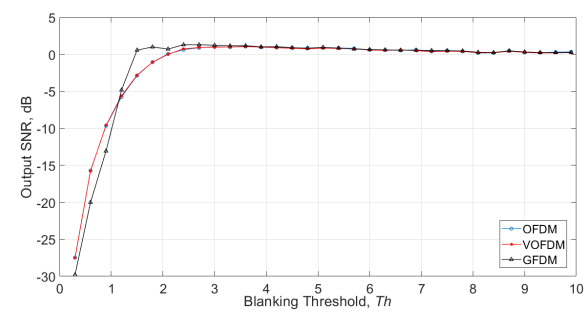

(c) $\lambda=0.5$.

Fig. 4: SNR with combined IN and NBI affected channel at $p=0.01$.

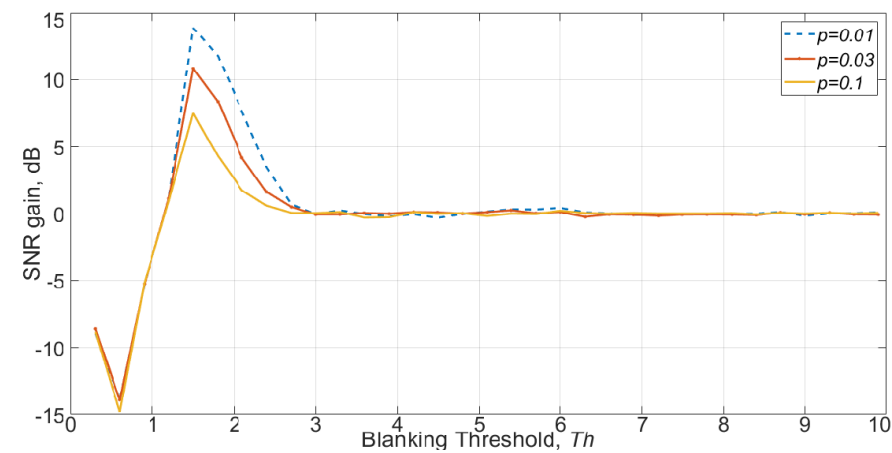

Fig. 5: SNR gain with only IN affected channels.

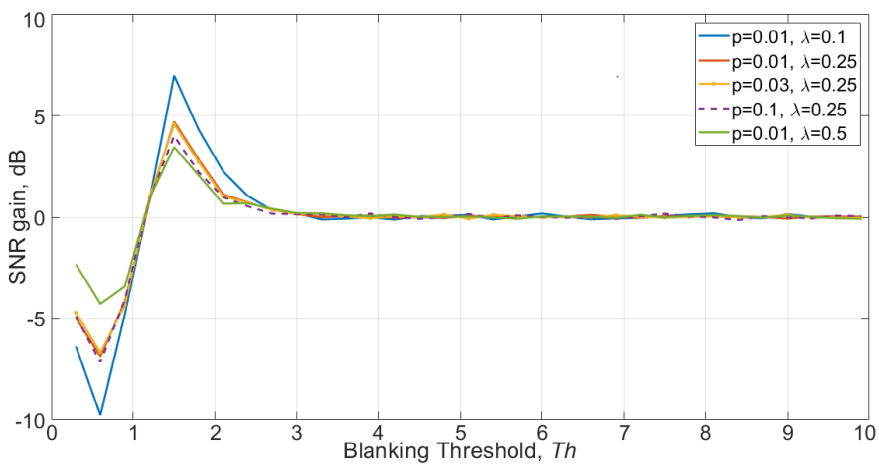

Fig. 6: SNR gain with IN and NBI affected channels.

other advanced techniques to reduce both IN and NBI may lead to further improvement. The results are varying with blanking threshold and best results are only achieved at an optimised threshold, which is different for OFDM and GFDM. The reason for this threshold difference can be considered as the result of difference in PAPR. The PAPR of OFDM is approximately 5 times the PAPR of GFDM. Therefore, better results of GFDM make it a suitable for $5 \mathrm{G}$ scenarios.

\section{REFERENCES}

[1] Y. Cai, Z. Qin, F. Cui, G.Y. Li and J.A. McCann, "Modulation and multiple access for 5G networks," IEEE Communications Surveys \& Tutorials, vol. 20, no. 1, pp. 629-646, Oct. 2017.

[2] N. Marchetti, M. I. Rahman, S. Kumar, and R. Prasad, "OFDM: Principles and Challenges," New Directions in Wireless Communications Research, pp. 29-62, 2009.

[3] X.-G. Xia, "Precoded and vector OFDM robust to channel spectral nulls and with reduced cyclic prefix length in single transmit antenna systems," IEEE Transactions on Communications, vol. 49, no. 8, pp. 1363-1374, Aug. 2001.

[4] G. Fettweis, M. Krondorf, and S. Bittner, "GFDM - Generalized Frequency Division Multiplexing," Proc. of IEEE $69^{\text {th }}$ Vehicular Technology Conference, 2009.

[5] G. Al-Juboori, E. Tsimbalo, A. Doufexi, and A. R. Nix, "A Comparison of OFDM and GFDM-Based MFSK Modulation Schemes for Robust IoT Applications," Proc. of IEEE 85 ${ }^{\text {th }}$ Vehicular Technology Conference, 2017.

[6] L. M. H. Shhab, A. Rizaner, A. H. Ulusoy, and H. Amca, "Impact of impulsive noise on millimeter wave cellular systems performance," Proc. of $10^{\text {th }}$ UK-Europe-China Workshop on Millimetre Waves and Terahertz. Technologies (UCMMT), 2017.

[7] Z. Ali, F. Ayaz, and C.-S. Park, "Optimized threshold calculation for blanking nonlinearity at OFDM receivers based on impulsive noise estimation," EURASIP Journal on Wireless Communications and Networking, vol. 2015, no. 1, Jul. 2015.

[8] F. Ayaz, K. Rabie, and B. Adebisi, "Analysis of Optimized Threshold with SLM based Blanking Non-Linearity for Impulsive Noise Reduction in Power Line Communication Systems," Proc. of $11^{\text {th }}$ International Symposium on Communication Systems, Networks and Digital Signal Processing (CSNDSP), 2018.

[9] A. G. Bolaji and T. Shongwe, "BPSK-OFDM versus QFSK-OFDM in Combating the Effects of Narrowband Interference and Impulsive Noise in Power Line Communication," Proc. of $11^{\text {th }}$ International Symposium on Communication Systems, Networks and Digital Signal Processing (CSNDSP), 2018. 